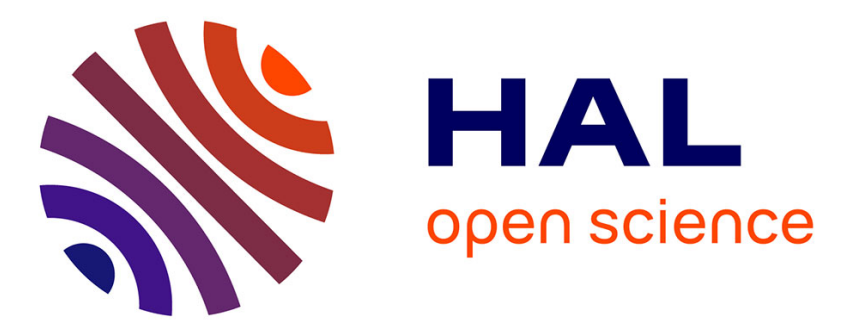

\title{
Complementary Communication Path for Energy Efficient On-Chip Optical Interconnects
}

\author{
Hui Li, Sébastien Le Beux, Yvain Thonnart, Ian O'Connor
}

\section{To cite this version:}

Hui Li, Sébastien Le Beux, Yvain Thonnart, Ian O'Connor. Complementary Communication Path for Energy Efficient On-Chip Optical Interconnects. 52th IEEE Design Automation Conference (DAC), Jun 2015, San francisco, United States. 10.1145/2744769.2744810 . hal-01243276

\section{HAL Id: hal-01243276 \\ https://hal.inria.fr/hal-01243276}

Submitted on 14 Dec 2015

HAL is a multi-disciplinary open access archive for the deposit and dissemination of scientific research documents, whether they are published or not. The documents may come from teaching and research institutions in France or abroad, or from public or private research centers.
L'archive ouverte pluridisciplinaire HAL, est destinée au dépôt et à la diffusion de documents scientifiques de niveau recherche, publiés ou non, émanant des établissements d'enseignement et de recherche français ou étrangers, des laboratoires publics ou privés. 


\title{
Complementary Communication Path for Energy Efficient On-Chip Optical Interconnects
}

\author{
Hui Li ${ }^{1}$, Sébastien Le Beux ${ }^{1 *}$, Yvain Thonnart ${ }^{2}{ }^{2}$, Ian O'Connor ${ }^{1}$ \\ ${ }^{1}$ Lyon Institute of Nanotechnology, INL-UMR5270, Ecole Centrale de Lyon, F-69134 Ecully, France \\ ${ }^{2}$ Univ. Grenoble Alpes, F-38000 Grenoble, France \\ ${ }^{3}$ CEA, LETI, MINATEC Campus, F-38054 Grenoble, France \\ hui.li@doctorant.ec-lyon.fr, sebastien.le-beux@ec-lyon.fr, yvain.thonnart@cea.fr, ian.oconnor@ec-lyon.fr
}

\begin{abstract}
Optical interconnects are considered to be one of the key solutions for future generation on-chip interconnects. However, energy efficiency is mainly limited by the losses incurred by the optical signals, which considerably reduces the optical power received by the photodetectors. In this paper we propose a differential transmission of the modulated signals, which contributes to improve the transmission of the optical signal power on the receiver side. With this approach, it is possible to reduce the input laser power and increase the energy efficiency of the optical communication. The approach is generic and can be applied to SWSR-, MWSR-, SWMR- and MWMR-like architectures.
\end{abstract}

\section{Categories and Subject Descriptors}

C.1.2 [Processor Architectures]: Multiple Data Stream Architectures (Multiprocessors) - Interconnection architectures (e.g., common bus, multiport memory, crossbar switch)

\section{General Terms}

Design

\section{Keywords}

Optical interconnect, Differential transmission

\section{INTRODUCTION}

Technology scaling, increased die sizes and die stacking are still promising to increase the processing density of advanced CMOS circuits. Yet, in order to push further the many-core integration, chip-level communication between processing clusters needs a deep restructuring to fit in power budgets. Silicon photonics is seen as a key enabling technology for highbandwidth communication across large chips or interposers, with limited power consumption and low footprint. Optical networks on chip (ONoC) were proposed as a scalable solution to interconnect a large number of processing cores. However, chiplevel optical communication in silicon waveguides incurs more losses than fiber-optic communication $(\mathrm{dB} / \mathrm{cm}$ vs. $\mathrm{dB} / \mathrm{km})$, which needs to carefully optimize the optical paths from the light

*Corresponding author. sources, so as to maintain high energy efficiency. A comprehensive work has been done on energy-efficient ONoC topologies, including MWMR- (Multiple Writer Multiple Reader [3]), MWSR- (Multiple Writer Single Reader [4]), SWMR(Single Writer Multiple Reader [5]) and SWSR- (Single Writer Single Reader [8]) like architectures.

A recurrent communication pattern in all these topologies is a serpentine-like structure, possibly opened at various points, crossing all the processing cores with one or more waveguides used to propagate modulated light on different wavelengths, with on-off keying modulation (light transmitted or absorbed). In this paper, evolving on this pattern, we propose to recover the absorbed light, to re-inject it in a complementary waveguide, and provide differential optical communication. With this approach, it is possible to reduce the input laser power and increase the energy efficiency of the optical communication. Our approach is generic and can be applied to a large panel of existing architectures.

Section 2 of this paper presents the basic communication structures for the proposed approach and their integration. Section 3 proposes an analytical model for the optical power budget evolution from the light sources to the optoelectronic receivers. And finally, Section 4 presents case studies on the application to MWMR, MWSR, SWMR, SWSR and generic architectures with the associated power savings.

\section{PROPOSED APPROACH}

In this section, we present an implementation of the proposed approach enabling the differential transmission of modulated data. The elementary structure is then introduced.

\subsection{Approach Overview}

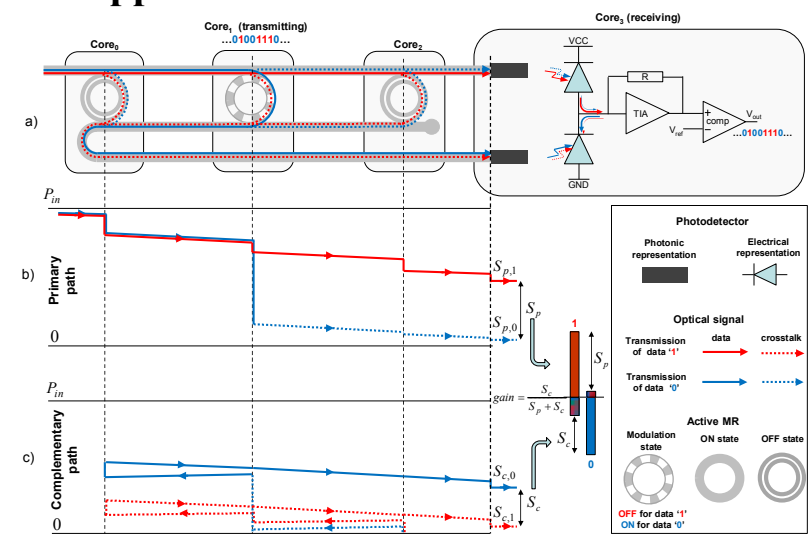

Figure 1. Transmission of data ' 1 ' and ' 0 ' through primary and complementary paths respectively.

Figure 1-a illustrates our approach using a MWSR-like architecture, assuming 3 Writers, one waveguide and a single wavelength (i.e. one MR per core). In the example, Core ${ }_{1}$ communicates with $\mathrm{Core}_{3}$. Hence, the MRs in $\mathrm{Core}_{0}$ and $\mathrm{Core}_{2}$ are 
turned OFF (i.e. at off-resonance) and the MR in Core $_{1}$ is in the modulation state, i.e. it switches from OFF state to $\mathrm{ON}$ state (i.e. at resonance) to transmit data ' 1 ' and ' 0 ' respectively.

\subsubsection{Primary Path: Transmission of Data '1'}

Data ' 1 ' leads to a MR in the OFF state to let the light pass through. Similarly to the related approaches, the light propagates through the so-called primary path: it crosses the modulator and continues propagating along the same waveguide until reaching the photodetector, as illustrated by the red arrow in Figure 1-a. The power of the optical signal decreases due to losses from waveguide propagation, crossed modulators and photodetector, as illustrated by the red line in Figure 1-b. The optical power received by the photodetector $\left(S_{p, 1}\right)$ must be high enough to ensure proper detection and photo-electronic conversion through the Trans-Impedance Amplifier (TIA). Conversely, the MR is turned $\mathrm{ON}$ for data ' 0 ' and diverts most of the light from the primary path, leading to a low optical power $\mathrm{S}_{\mathrm{p}, 0}$ at the photodetector. While the sensitivity of the photodetector (of the order of $-20 \mathrm{dBm}$ [1]) is a key parameter for energy-efficient optical interconnect, the actual received power is usually higher to distinguish data ' 1 ' $\left(\mathrm{S}_{\mathrm{p}, 1}\right)$ from data ' 0 ' $\left(\mathrm{S}_{\mathrm{p}, 0}\right)$ and to ensure high enough signal-to-noise ratio (SNR) in the worst-case scenario. It is well known that optical interconnects are sensitive to crosstalk and variations in both the fabrication process and local temperature. We define $S_{p}$ as the difference between the signal power received when transmitting ' 1 ' and ' 0 ' in the primary path.

\subsubsection{Complementary Path: Transmission of Data '0'}

When the MR is in the ON state to transmit data ' 0 ' in the primary path, instead of "absorbing" the light as in related approaches, we propose to drop the light into a secondary path, the so-called complementary path, as illustrated by the blue arrow in Figure 1-a. In this example, it is composed of a waveguide propagating the light towards a second photodetector receiving $\mathrm{S}_{\mathrm{c}, 0}$. This complementary structure allows the differential transmission of the data, which contributes to increasing the detected signal power and ultimately reduces the laser power consumption. The signal propagating in the complementary path experiences some power increase due to the crosstalk signals dropped from the primary path (i.e. dotted line arrows from Core $\mathrm{C}_{0}$ and $\mathrm{Core}_{2}$ in the figure). This positive effect is partially counteracted by the increase of $\mathrm{S}_{\mathrm{c}, 1}$, the crosstalk level in the complementary path when a ' 1 ' is transmitted (i.e. dashed red line), as illustrated in Figure 1-c. $\mathrm{S}_{\mathrm{c}}$ is the difference between the signal power received in the complementary path when transmitting ' 0 ' and ' 1 '. This additional received power enables the value of the transmitted data to be better distinguished thanks to a higher dynamic of the signals. Getting $\mathrm{S}_{\mathrm{p}}+\mathrm{S}_{\mathrm{c}}$ instead of $\mathrm{S}_{\mathrm{p}}$, it is thus possible to reduce the input laser power by a factor of $\mathrm{S}_{\mathrm{p}} /\left(\mathrm{S}_{\mathrm{p}}+\mathrm{S}_{\mathrm{c}}\right)$, i.e. with an energy efficiency gain of $\mathrm{S}_{\mathrm{c}} /\left(\mathrm{S}_{\mathrm{p}}+\mathrm{S}_{\mathrm{c}}\right)$.

\subsubsection{Micro-Architecture of the Receiver}

The addition of a complementary path to the conventional transmission structure creates the need for a modified receiver, which will benefit from the differential transmission to improve the optical signal quality and allow working at lower laser input power. To do so, the photocurrents provided by the photodiodes on the primary and complementary paths are substracted by a simple series connection of the photodiodes between supply and ground, the difference current being fed to a conventional TIA, as depicted in Figure 1-a.

The analytical models presented hereafter consider a linear conversion from the photocurrents to the TIA output, which actually means the signal is considered to propagate without alteration from the optical subsystem to the electrical subsystem.

\subsection{Elementary Blocks for Signal Analysis}
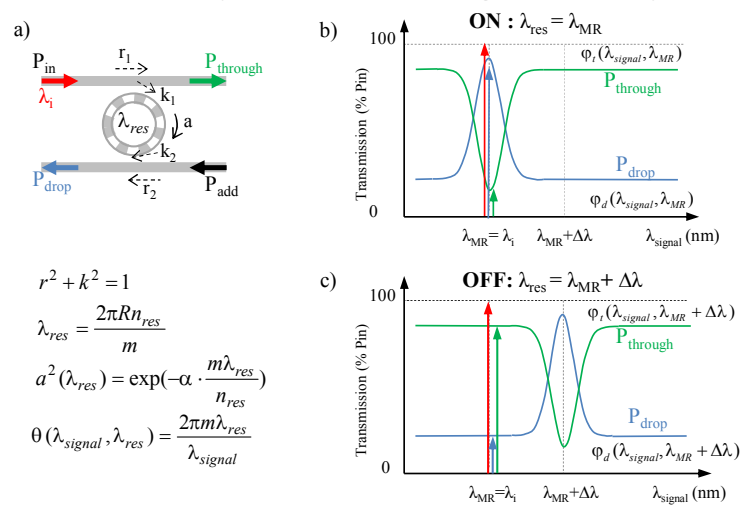

Figure 2. MR model: a) device geometry, signal transmission on through port and drop port in b) ON state and c) OFF state.

Table 1: Transmission parameters.

\begin{tabular}{|c|c|c|}
\hline Parameter & Description & Unit \\
\hline $\mathrm{r}_{1}, \mathrm{r}_{2}$ & Self-coupling coefficient & \\
\hline $\mathrm{k}_{1}, \mathrm{k}_{2}$ & Cross-coupling coefficient & \\
\hline $\mathrm{R}$ & MR radius & $\mu \mathrm{m}$ \\
\hline $\mathrm{n}_{\text {res }}$ & $\begin{array}{l}\text { Effective refractive index of MR (varies } \\
\text { with applied voltage, device geometry and } \\
\text { ambient temperature) }\end{array}$ & \\
\hline $\mathrm{m}$ & Resonant mode number of MR & \\
\hline$\lambda_{\text {res }}$ & MR resonant wavelength & \\
\hline$\lambda_{\text {signal }}$ & Signal wavelength & $\mathrm{nm}$ \\
\hline$\Delta \lambda$ & $\begin{array}{l}\text { MR wavelength between } \mathrm{ON} \text { and OFF } \\
\text { states }\end{array}$ & $\mathrm{nm}$ \\
\hline$\alpha$ & Power attenuation coefficient & $\mathrm{dB} / \mathrm{cm}$ \\
\hline $\mathrm{a}\left(\lambda_{\text {res }}\right)$ & Single-pass amplitude transmission & \\
\hline$\theta\left(\lambda_{\text {signal }}, \lambda_{\text {res }}\right)$ & Single-pass phase shift & \\
\hline $\mathrm{L}_{\mathrm{b}}$ & $180^{\circ}$ bending loss under $40 \mu \mathrm{m}$ radius & $\mathrm{dB}$ \\
\hline
\end{tabular}

$$
\begin{aligned}
& \varphi_{t}\left(\lambda_{\text {signal }}, \lambda_{\text {res }}\right)=\frac{a^{2}\left(\lambda_{\text {res }}\right) r_{2}^{2}-2 a\left(\lambda_{\text {res }}\right) r_{1} r_{2} \cos \left[\theta\left(\lambda_{\text {signal }}, \lambda_{\text {res }}\right)\right]+r_{1}^{2}}{1-2 a\left(\lambda_{\text {res }}\right) r_{1} r_{2} \cos \left[\theta\left(\lambda_{\text {signal }}, \lambda_{\text {res }}\right)\right]+\left[a\left(\lambda_{\text {res }}\right) r_{1} r_{2}\right]^{2}} \\
& \varphi_{d}\left(\lambda_{\text {signal }}, \lambda_{\text {res }}\right)=\frac{a\left(\lambda_{\text {res }}\right)\left(1-r_{1}^{2}\right)\left(1-r_{2}^{2}\right)}{1-2 a\left(\lambda_{\text {res }}\right) r_{1} r_{2} \cos \left[\theta\left(\lambda_{\text {signal }}, \lambda_{\text {res }}\right)\right]+\left[a\left(\lambda_{\text {res }}\right) r_{1} r_{2}\right]^{2}}
\end{aligned}
$$

The improvements induced by the complementary path depend on the transmission of the optical signals in the through port and the drop port of a MR (Figure 2-a). A MR is characterized by a resonant wavelength $\left(\lambda_{\text {res }}\right)$ in the ON state, i.e. the input signals at $\lambda_{\text {signal }}=\lambda_{\text {res }}$ are redirected to the drop port. In the OFF state, the resonant wavelength drifts by $\Delta \lambda$, i.e. the input signals at $\lambda_{\text {signal }} \neq \lambda_{\text {res }}$ will continue propagating in the same waveguide. Figure 2-b-c illustrates the transmissions on through port and drop port (i.e. $\varphi_{t}$ and $\varphi_{d}$ ) in ON and OFF states respectively. The actual transmissions depend on the device geometry (i.e. ring radius, $\mathrm{R}$ ), the self(cross)-coupling coefficient (i.e. $r_{1}, r_{2}, k_{1}$ and $k_{2}$ ), the power attenuation coefficient $\alpha$, and the single-pass phase shift $\theta\left(\lambda_{\text {signal }}, \lambda_{\text {res }}\right)$. Table 1 summarizes the parameters and Equations(1) and(2) give the transmission $\varphi_{t}$ and

$\varphi_{d}$ extracted from [2].

Figure 3 illustrates the elementary optical writing and reading structures, respectively named writer $\mathrm{w}_{\mathrm{j}}$ and reader $\mathrm{r}_{\mathrm{j}}$. In the writer, the modulation of data ' 1 ' leads to the propagation of the signal at $\lambda_{j}$ on the through port (Figure $3-a$ ). The losses of the 
signal are thus expected to be the same as in traditional architectures. If data ' 0 ' is modulated, the signal at $\lambda_{\mathrm{j}}$ is strongly attenuated on the through port and is mainly redirected to the drop port. The signal then i) propagates on a waveguide following the backward-direction and ii) is redirected to the forward-direction using a U-shaped waveguide (Figure 3-b). In order to reduce the waveguide bending losses $\mathrm{L}_{\mathrm{b}}$, a $40 \mu \mathrm{m}$ radius is considered, which leads to $-0.025 \mathrm{~dB}$ losses based on equations in Figure 2-a (Figure 3 is not at the right scale: the radius of the ring is around $1-10 \mu \mathrm{m}$ and is thus smaller than the U-shaped waveguide).
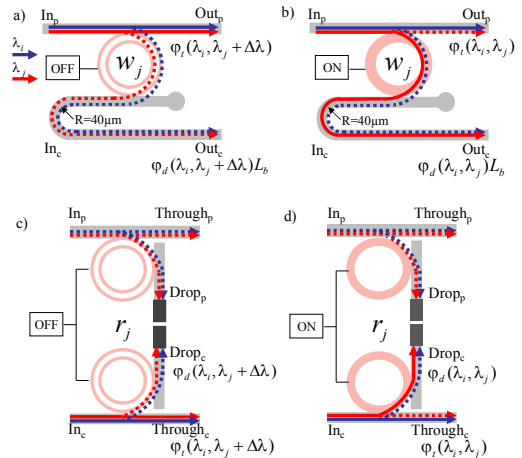

Figure 3. The writer $w_{j}$ includes an MR that a) let the signals at $\lambda_{j}$ continue propagating on the primary path or b) drops the resonating signal toward the complementary path. The reader $r_{j}$ is symmetric and includes two MRs allowing to a) let signals continue propagating or d) filter them for photodetection.

The aim of $r_{j}$ is to filter the same wavelength on both primary and complementary paths, as illustrated in Figure 3c-d. For this purpose, the traditional reader structure is duplicated and the resulting MR pair is controlled by a common signal (note that there are no MRs in the reader structure in Figure 1-a since a single wavelength was considered). These additional MRs lead to the actual cost of the proposed approach. In the result section, we will compare the reduction of the laser power to the increase of the area.

\section{ANALYTICAL MODEL}

\subsection{General MWMR Model}

Figure 4 illustrates the MWMR architecture we are considering. It is composed of $\mathrm{N}_{\mathrm{W}}$ Writers and $\mathrm{N}_{\mathrm{R}}$ Readers (the general case allows $\mathrm{N}_{\mathrm{W}} \neq \mathrm{N}_{\mathrm{R}}$ ). Each waveguide pair of the network (a single pair in the figure) performs 2 round trips to successively cross the Writers and the Readers. CW lasers inject optical power in each primary path. We assume that $\mathrm{N}_{\mathrm{WL}}$ wavelengths are used to modulate the signal (i.e., $\lambda_{0}, \lambda_{1}, \ldots, \lambda_{\mathrm{NWL}-1}$ ). The modulation of the signals occurs in $\mathrm{W}_{\mathrm{S}}$, the Writer part of the source core Core (note that $\mathrm{W}_{\mathrm{S}}$ is composed of $\mathrm{w}_{0}, \mathrm{w}_{1}, \ldots, \mathrm{w}_{\mathrm{NWL}-1}$ ), and experiences the detection in the $\mathrm{R}_{\mathrm{T}}$, the Reader part of the target core Core $\mathrm{T}_{\mathrm{T}}$ $\left(\mathrm{R}_{\mathrm{T}}\right.$ is composed of $\left.\mathrm{r}_{0}, \mathrm{r}_{1}, \ldots, \mathrm{r}_{\mathrm{NWL}-1}\right)$. The aim of the analytical model is to evaluate the laser power reduction achievable with the use of the complementary path. For this purpose, we distinguish the signal transmission in the set of all writers and the set of all receivers.

\subsubsection{Transmission in the Writers}

The losses experienced by the optical signal crossing the writers will depend on i) the modulated data $d_{j}$ (i.e. ' 0 ' or ' 1 ') and ii) the core position where the modulation takes place, $W_{S}$. In order to distinguish the transmissions $\mathrm{T}$ of a signal at wavelength $\lambda_{\mathrm{j}}$ in the primary path and the complementary path, we define $T_{w, p, W_{S}, d_{j}}[j]$ and $T_{w, c, W_{S}, d_{j}}[j]$ respectively. In this notation, $\mathrm{d}_{\mathrm{j}}$ is the value of the modulated data (' 0 ' or ' 1 ') at $\lambda_{j}$, which is important to take into account since it determines the optical path. These transmissions allow the power level of the optical signal to be evaluated before entering the set of Readers (in the second waveguide round trip). For the sake of clarity, the formulas for $T_{w}$ are given in Section 6.

\subsubsection{Transmission in the Readers}

Due to a symmetric micro-architecture and control circuitry of the reader structure, the signals in primary and complementary paths experience the same transmission to reach $\mathrm{R}_{\mathrm{T}}$, and are expressed as $T_{r, R_{T}, j}[j]$. After crossing intermediate Reader cores $\left(\mathrm{R}_{0} \ldots \mathrm{R}_{\mathrm{T}-1}\right)$, the signals at wavelength $\lambda_{\mathrm{j}}$ reach $\mathrm{R}_{\mathrm{T}}$ where they are dropped by the appropriate filters toward photodetectors. The formula for $T_{r}$ is also given in Section 6.

\subsubsection{Received Signal Power}

In order to estimate the gain induced by the complementary path, we define $S_{p, W_{S}, R_{T}, j, d_{j}}$ and $S_{c, W_{S}, R_{T}, j, d_{j}}$ as the signals modulated in $\mathrm{W}_{\mathrm{S}}$ and received by the $\mathrm{j}^{\text {th }}$ photodetectors in $\mathrm{R}_{\mathrm{T}}$ on primary and complementary paths respectively. The four cases are formally defined as:

$$
\begin{aligned}
& S_{p, W_{S}, R_{T}, j, 0}=T_{w, p, W_{S}, 0}[j] \cdot T_{r, R_{T}, j}[j] \cdot P_{i n}[j] \\
& S_{p, W_{S}, R_{T}, j, 1}=T_{w, p, W_{S}, 1}[j] \cdot T_{r, R_{T}, j}[j] \cdot P_{i n}[j] \\
& S_{c, W_{S}, R_{T}, j, 0}=T_{w, c, W_{S}, 0}[j] \cdot T_{r, R_{T}, j}[j] \cdot P_{i n}[j] \\
& S_{c, W_{S}, R_{T}, j, 1}=T_{w, c, W_{S}, 1}[j] \cdot T_{r, R_{T}, j}[j] \cdot P_{i n}[j]
\end{aligned}
$$

where $\mathrm{P}_{\text {in }}[\mathrm{j}]$ is the laser power coupled into the waveguide at $\lambda_{\mathrm{j}} . S_{p, W_{S}, R_{T}, j}$ (resp. $S_{c, W_{S}, R_{T}, j}$ ) corresponds to the difference between the received signal powers for $\mathrm{d}_{\mathrm{j}}={ }^{\prime} 1$ ' and $\mathrm{d}_{\mathrm{j}}={ }^{\prime} 0$ ' (resp. ' 0 ' and ' 1 '). From this, we obtain the signal transmission improvement for communication $\mathrm{W}_{\mathrm{S}} \rightarrow \mathrm{R}_{\mathrm{T}}$, which is defined as

$$
\frac{S_{c, W_{S}, R_{T}, j}}{S_{p, W_{S}, R_{T}, j}+S_{c, W_{S}, R_{T}, j}}
$$

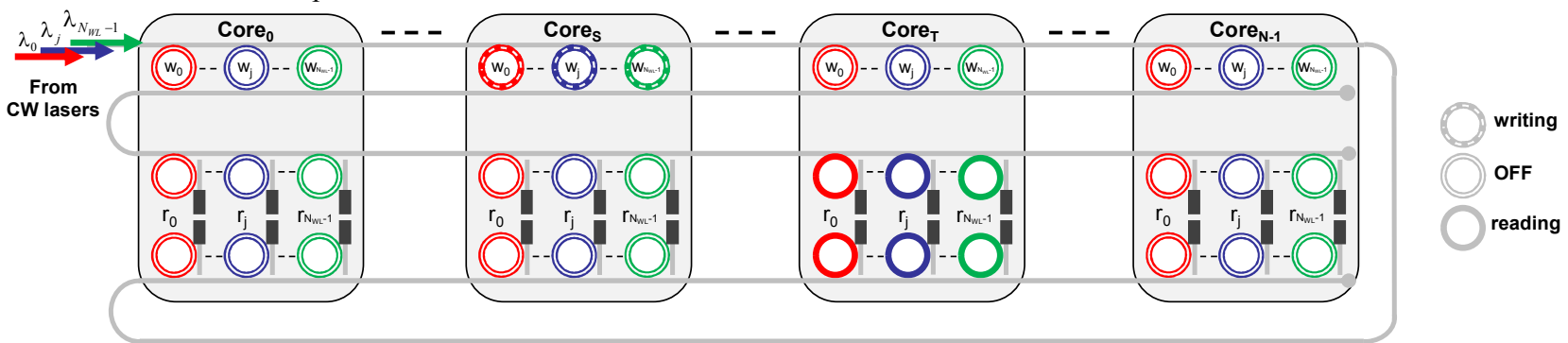

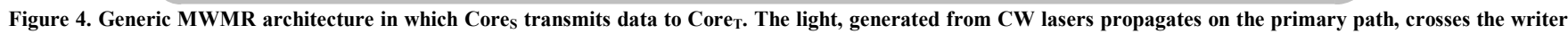

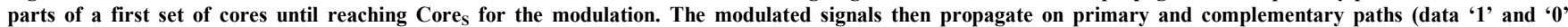
respectively), cross a second set of writers, a set of readers until reaching the photodetectors of Core T. $_{\text {. }}$ 
By considering the worst case transmission improvement among the possible communication pairs, the laser power reduction is estimated.

For the sake of clarity, the equations described above do not take into account the inter-channel crosstalk (which occurs due to the signals modulated at other wavelengths). We refer to Section 6 for the general case in which inter-channel crosstalk is considered. All the analyses in Section 4 rely on the models taking into account the inter-channel crosstalk.

\subsection{Depopulated Models}

This MWMR model allows the gain of our approach to be applied in network architectures such as Flexishare [3]. In addition, the analytical model can be adapted to other types of network such as MWSR, SWMR and SWSR. The model in Section 3.1 is adapted based on the implementation of the complementary path structure in these networks, which is illustrated in Figure 5.
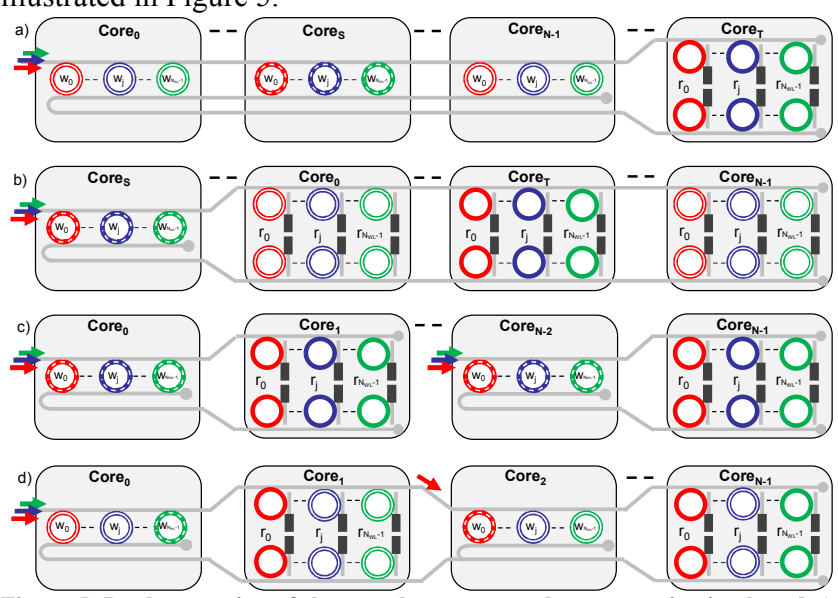

Figure 5. Implementation of the complementary path structure in ring-based a) MWSR, b) SWMR and c) SWSR like networks. Part d) illustrates its implementation on sectioned waveguides based networks.

Figure 5-a illustrates a MWSR architecture which is obtained by considering a single target core per waveguide. In such network architecture, each core is reachable through a set of dedicated waveguides. Among others, MPNoC [5], Corona [4] and Clos [9] rely on MWSR. On the opposite, SWMR-like architectures (Figure 5-b) imply a single source core per waveguide, i.e. the arbitration occurs in the Readers. Networks such as Firefly [7], LumiNOC [12], ATAC unicast channel [6] implement SWMR. Finally, SWSR-like architecture is obtained by allocating, at design time, dedicated wavelengths, i.e. no arbitration is required. The immediate implementation of a SWSR network leads to a single writing core and a single reading core per waveguide. It thus implies dedicated waveguides for

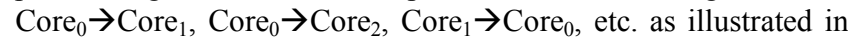
Figure 5-c. For all these scenarios (Figure 5-a-b-c), we have derived the analytical model from Section 6, taking into account the (possibly different) number of Writers (x) and Readers (y), i.e. which is denoted as $x$ WyR. In Section 4, we evaluate the gain of the complementary path in these architectures.

Our approach suits well with serpentine layout-based networks since it does not add complexity in the layout and does not introduce waveguide crossing. Hence, it can also be applied to networks relying on sectioned waveguides (Figure 5-d) such as ORNoC [8], Chameleon [10] and SUOR [11]. The improvement evaluation of the complementary path in these networks is remained as future work. While it could also be applied to other topologies such as Mesh, Torus [13] and Multi-Stage, it would need to duplicate all rings used as routers, and would also introduce many waveguide crossings that will, in turn, reduce the signal power on primary and complementary paths. For this reason, these topologies options are not considered.

\subsection{Simulation Model}

To investigate the dynamic behavior of our proposal, and analyze the optical power budget along the complete transmission path, compact models of the MRs and photodetectors were encoded in Verilog-A. The MR models are based on the equations of $\varphi_{\mathrm{t}}$ and $\varphi_{\mathrm{d}}$ as described previously, including parasitic resistances and capacitances for the electrical modulation, and by modeling the refractive index changes (real index and imaginary part for absorption) due to the modulation voltage. The photodiode models are based on a conventional diode equation including dark current along with a linear responsivity of the photocurrent (in $\mathrm{A} / \mathrm{W}$ ).

\section{CASE STUDY}

\subsection{MWMR}

Modulation using MRs is usually done in two ways. One way is based on doping the ring to form a $\mathrm{P}-\mathrm{N}$ junction at the ring level, such that reverse biasing will change the refractive index through carrier depletion. The other way is to leave the ring undoped, with $\mathrm{P}$ and $\mathrm{N}$ doping on either side, so as to create a P-I$\mathrm{N}$ junction, which will change the refractive index by carrier injection when forward biased. PN modulators are known to have a faster switching time [14] than PIN modulators for a given bias voltage, which makes them an interesting option for high-speed modulation. Nevertheless, the high absorption in the rings due to the cloud of carriers at the junction leads to lower transmission ratios toward the through and drop ports.

Table 2. Parameters and values for the writer and the reader.

\begin{tabular}{|l|c|c|}
\hline \multirow{2}{*}{ Parameter } & PN & Value \\
\cline { 2 - 3 } & 0.992 & 0.998 \\
\hline $\mathrm{r}_{1}{ }^{2}$ & 0.996 & 0.998 \\
\hline $\mathrm{r}_{2}{ }^{2}$ & $15 \mathrm{~dB} / \mathrm{cm}$ & $2 \mathrm{~dB} / \mathrm{cm}$ \\
\hline$\alpha$ & $0.09 \mathrm{~nm}($ under $2.5 \mathrm{~V})$ & $0.16 \mathrm{~nm}($ under $2 \mathrm{~V})$ \\
\hline$\Delta \lambda$ & $\sim 59 \mathrm{~nm}$ (allowing 72 channels) \\
\hline FSR & \multicolumn{2}{|c|}{$\sim 2.55$} \\
\hline $\mathrm{n}_{\mathrm{MR}}$ & \multicolumn{2}{|c|}{17} \\
\hline $\mathrm{m}$ & \multicolumn{2}{|c|}{$-0.025 \mathrm{~dB}$} \\
\hline $\mathrm{L}_{\mathrm{b}}$ & \multicolumn{2}{|c|}{} \\
\hline
\end{tabular}

Table 2 presents the parameter values for both types of rings for transmission covering the complete C-band (around $1550 \mathrm{~nm}$ ). The 72 dense wavelength-division-multiplexing channels (DWDM) of the C-band require non-interference of higher resonance orders between rings. Therefore, a Free-Spectral-Range (FSR) above $58 \mathrm{~nm}$ is targeted, resulting in resonance at order 17 in rings of around $1.6 \mu \mathrm{m}$ of radius. The coupling coefficients of the rings to the waveguides are chosen in order to optimize the contrast between ' 1 ' and ' 0 ' at the through and drop ports.

In order to minimize the losses in the set of receivers, PIN MRs are preferably used on the receiver side: indeed, they have lower losses, and are configured at the granularity of a packet, i.e. with a lower switching frequency than the modulators. The choice of writers, conversely, is not obvious, and will be studied in the next paragraph. We analyze the benefits of the complementary path in a 4W4R network with 4 wavelengths.

The 4W4R network has been captured as a schematic in the Cadence Virtuoso editor, and the resulting netlist has been fed to Mentor Graphics Eldo simulator, using the compact models of the 
optical elements that were introduced in Section 3.3. Two different netlists were generated, using PN and PIN modulators. This allowed extracting the evolution of the optical power budget along the primary and complementary paths. The communication from $\mathrm{C}_{0}$ to $\mathrm{C}_{3}$ is presented in Figure 6 .

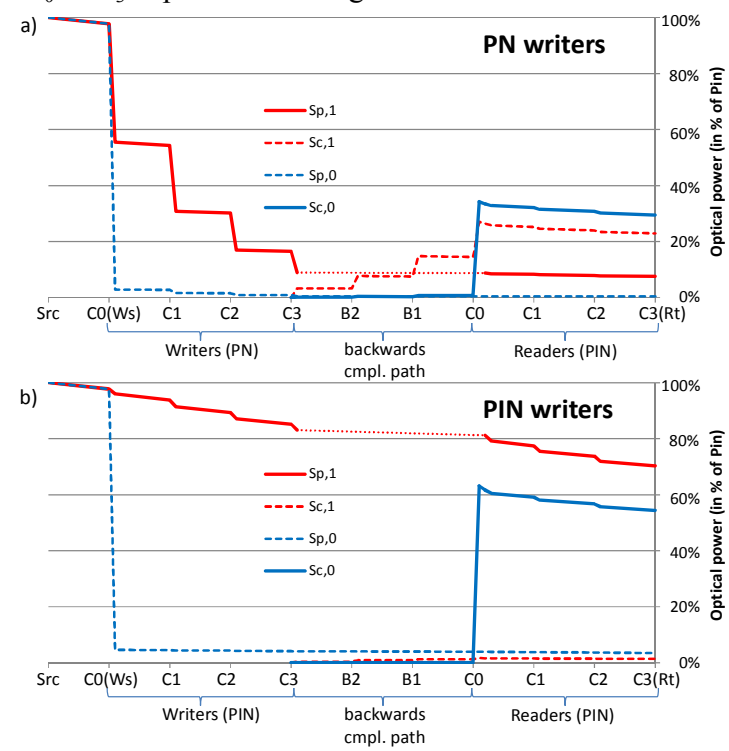

Figure 6. Evolution of the optical power budget along the primary and complementary paths for a) PN writers, and b) PIN writers.

Using PN modulators as writers (Figure 6-a), the attenuation is relatively important, and the primary signal rapidly decreases along the writers chain. Conversely, the complementary signal passes by a single $\mathrm{PN}$ ring at Core $_{0}$ where it is deflected to the complementary path. Hence, $\mathrm{S}_{\mathrm{c}, 0}$ is eventually higher than $\mathrm{S}_{\mathrm{p}, 1}$. Moreover, the optical leakage to the drop port in through mode is still important, and these contributions are added along the path, up to a level $\mathrm{S}_{\mathrm{c}, 1}$.

As this behavior is not acceptable, it is needed to consider PIN rings for modulation, which thanks to a higher shift in resonance $(\Delta \lambda)$ with the modulation voltage allows for a much better transmission in through mode and reduced optical leakage to the drop port. Figure 6-b presents the results with PIN modulators for the same communication as before, which show a much better separation between ' 0 ' and ' 1 ' in both the primary and complementary paths. The drawback is that to compensate for the slower bandwidth of these PIN modulators, the modulation requires complex drivers with pre-emphasis. This leads to increased area and power consumption in the CMOS drivers [14]. However, this electrical power consumption increase remains negligible with respect to the optical power consumption of the lasers.

We computed values $S_{p}$ and $S_{c}$ for all the communication pairs in $4 \mathrm{~W} 4 \mathrm{R}$ architecture. These values fit within $3 \%$ of the previous simulation. The difference is a cumulative error in through mode due to ring radius variation across WDM channels. $\mathrm{C}_{3} \rightarrow \mathrm{C}_{2}$ is the communication showing the smallest signal transmission improvement (44\%); it occurs when the last Writer communicates with the last Reader.

\subsection{Large Scale MWMR-like Architectures}

We evaluate the impact of the complementary path on MWMR architectures including up to 16 Writers and 16 Readers. It is worth noticing that, assuming a total connectivity between the cores and a serpentine layout, using only (many) SWSR rings will lead to the same waveguide length as with only a (single) $16 \mathrm{~W} 16 \mathrm{R}$ ring. In all our results, we assume $11 \mathrm{~dB}$ propagation losses in each waveguide, which corresponds to a total $11 \mathrm{~cm}$ wire length under typical $1 \mathrm{~dB} / \mathrm{cm}$ losses [3]. We also assume $-20 \mathrm{dBm}$ [1] for the photodetectors sensitivity, i.e. the laser input power $P_{\text {in }}$ should be adjusted to maintain more than $10 \mu \mathrm{W}$ optical power on the photodetectors. Figure 7-a gives the possible laser power reduction given this constraint for the worst-case communication scenario.

$$
\text { a) Laser power reduction wrt. traditional method }
$$
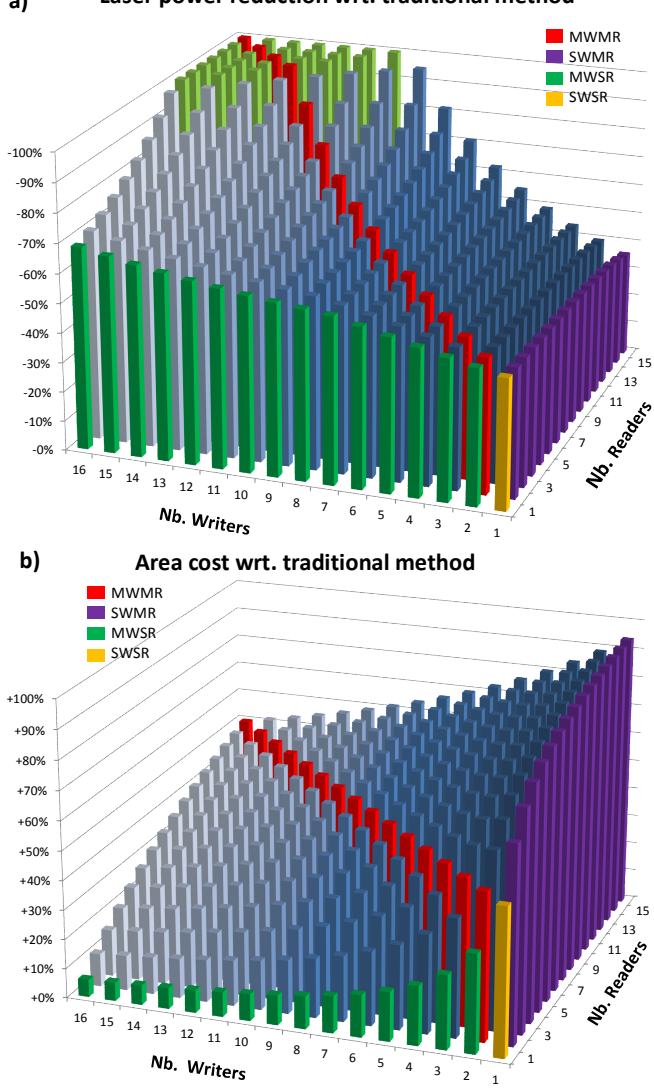

Figure 7. Impact of the complementary path on a) the laser power reduction and b) the area cost of the optical interconnect.

Our approach leads to some area overhead: in addition to the MRs required to implement the primary path $\left(A_{p}\right)$, additional MRs are required for the complementary path $\left(A_{c}\right)$. Figure 7-b represents the area cost (i.e. $A_{c} / A_{p}$ ).

Results show that the SWMR-like networks (purple color) allow around $40 \%$ laser power reduction independently from the number of Readers, as the losses are identical on both paths in the set of readers. However, the area cost considerably increases and reaches $+90 \%$ for SW16R due to doubling the MRs in all readers. We observe the opposite trend for MWSR-like architectures (dark green color): the more the Writers, the higher the power improvement and the lower the area cost. Actually, the complementary path eventually brings more power than the primary path, as the waveguide leads to fewer losses than MRs. For $16 \mathrm{WSR}$ case, power is reduced by $68 \%$, while area is only increased by $5 \%$ respectively. For MWMR-like networks (in red), power reduction is even more important while the area cost is constant. In a nutshell, our approach leads to the highest improvement for networks favoring writer aggregation. Experiments also demonstrate that, if we also bound the laser power possibly injected in the circuit (here $600 \mu \mathrm{W}$ ), the 
complementary path allows increasing the scale of the network by detecting signals not detectable in the primary path only (highlighted in light green, for 10-16 cores).

\section{CONCLUSION}

Differential optical communication in ONoCs has been demonstrated to lead to large savings in laser power for most ringbased topologies, sometimes with gains of much more than the intuitive factor of 2, thanks to reduced losses along the complementary path. MWSR communication shows a power divided by 3 for only $5 \%$ overhead. For multiple-Writer schemes, the MR overhead is always below $50 \%$. Actual area overhead is even smaller for 3D-stacked CMOS on photonics, as receiver signals are shared, and overhead is due to $3 \mathrm{D}$-interconnections. The proposed approach has been checked against simulations with compact VerilogA models. Complete electro-optical design of specific receivers and possible noise sensitivity reduction will be investigated in future works.

\section{APPENDIX: ANALYTICAL MODEL}

In this appendix, we consider communication between Writer $W_{S}$ and Receiver $R_{T}$. The received signal power on $r_{j}$ (i.e. the reader of $R_{T}$ at wavelength $\lambda_{j}$ ) depends on $d_{i}$ and $d_{j}$, the data modulated at $\lambda_{\mathrm{i}}$ (received power at $\lambda_{\mathrm{j}}$ is crosstalk) and $\lambda_{\mathrm{j}}$ respectively. They are defined by $S_{p, W_{S}, R_{T}, j, d_{j}}$ and $S_{c, W_{S}, R_{T}, j, d_{j}}$ which are formalized in Equations 5-a and 5-b respectively.

$$
\begin{aligned}
S_{p, W_{S}, R_{T}, j, d_{j}} & =\sum_{i=0}^{N_{W L}-1}\left(T_{w, p, W_{S}, d_{i}}[i] \cdot T_{r, R_{T}, j}[i] \cdot P_{i n}[i]\right) \\
S_{c, W_{S}, R_{T}, j, d_{j}} & =\sum_{i=0}^{N_{W L}-1}\left(T_{w, c, W_{S}, d_{i}}[i] \cdot T_{r, R_{T}, j}[i] \cdot P_{i n}[i]\right)
\end{aligned}
$$

They depend on:

- $T_{w, p, W_{S}, d_{i}}[i]$ (Eq. 6), transmission in the primary path of the writer parts, which is the same with that in traditional architectures.

- $\quad T_{w, c, W_{S}, d_{i}}[i]$ (Eq. 7), transmission in the complementary path of the writer parts. It is divided into: i) the transmission before the modulation, ii) the transmission from primary to complementary path where the modulation occurs and iii) the transmission after the modulation.

- $\quad T_{r, R_{T}, j}[i]$ (Eq. 8), transmission in the reader parts to reach the reading core. The transmission of signals at $\lambda_{\mathrm{i}}$ across the intermediate Readers and dropped by $\mathrm{R}_{\mathrm{T}}$ is defined.

In these equations, $L_{p}$ is the propagation loss $(-1 \mathrm{~dB} / \mathrm{cm}$ in the results section). $1_{w, \text { total }}[\mathrm{i}]$ and $1_{\mathrm{r}, \text { total }}[\mathrm{i}]$ (resp. $\mathrm{N}_{\mathrm{w}, \mathrm{b}}[\mathrm{i}]$ and $\mathrm{N}_{\mathrm{r}, \mathrm{b}}[\mathrm{i}]$ ) are the total waveguide length (resp. number of waveguide bends) experienced by signal at $\lambda_{\mathrm{i}}$ in Writers (in primary path) and Readers parts. Regarding the waveguide length experienced by signal at $\lambda_{\mathrm{i}}$ coupled from the primary path into the complementary path, we define it as $1_{v}[i]$, where $v$ is the considered Writer (i.e. $\mathrm{v}=\mathrm{Ws}$ when considering the modulating Writer).

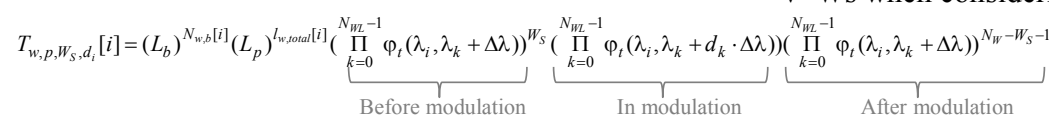

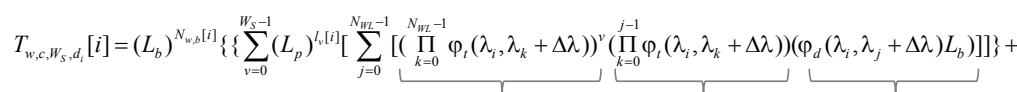

Before Writer v Before $w_{j} \quad$ Dropped by $w_{j}$

$$
\text { Before modulation }
$$

$$
\begin{aligned}
& \left\{\left(L_{p}\right)^{I_{W}[i]}[\underbrace{\sum_{W_{L}-1}}_{j=0}[\underbrace{\left.\sum_{\mathrm{S}}^{\sum_{k=0}-1} \varphi_{t}\left(\lambda_{i}, \lambda_{k}+\Delta \lambda\right)\right)^{W_{S}}}_{\text {Before Writer W }} \underbrace{\underbrace{j-1}_{k=0} \varphi_{t}\left(\lambda_{i}, \lambda_{k}+d_{k} \cdot \Delta \lambda\right))}_{\text {In modulation }} \underbrace{\left.\left.\left.\left(\varphi_{d}\left(\lambda_{i}, \lambda_{j}+d_{j} \cdot \Delta \lambda\right) L_{b}\right)\right]\right]\right\}}_{\text {Before } \mathrm{w}_{\mathrm{j}}}+\right. \\
& \left.\left\{\sum_{v=W_{S}+1}^{N_{y}-1}\left(L_{p}\right)^{l_{v}[i]}\left[\sum_{j=0}^{N_{v z}-1}\left[(\underbrace{\prod_{W L}-1}_{k=0} \varphi_{t}\left(\lambda_{i}, \lambda_{k}+\Delta \lambda\right))^{v-1}\left(\prod_{k=0}^{N_{W L}-1} \varphi_{t}\left(\lambda_{i}, \lambda_{k}+d_{k} \cdot \Delta \lambda\right)\right)\left(\prod_{k=0}^{j-1} \varphi_{t}\left(\lambda_{i}, \lambda_{k}+\Delta \lambda\right)\right)(\underbrace{}_{d}\left(\lambda_{i}, \lambda_{j}+\Delta \lambda\right) L_{b})\right]\right]\right\}\right\} \\
& \text { Before Writer v } \\
& \text { Before w } \\
& \text { Dropped by } \mathrm{w}_{\mathrm{j}}
\end{aligned}
$$

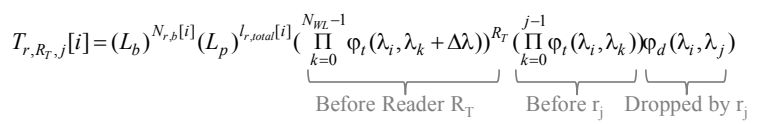

\section{REFERENCES}

[1] Aleksandr Biberman, et al. "Photonic Network-on-Chip Architectures Using Multilayer Deposited Silicon Materials for High-Performance Chip Multiprocessors," ACM Journal on Emerging Technologies in Computing Systems, 7 (2), 7:1-7:25, 2011.

[2] Wim Bogaerts, et al., "Silicon microring resonators," Laser\&Photonics Reviews, 6(1), 47-73, 2012.

[3] Yan Pan, John Kim and Gokhan Memik, "FlexiShare: Channel Sharing for an Energy-Efficient Nanophotonic Crossbar,” In HPCA, 2010.

[4] Dana Vantrease, et al., "Corona: System Implications of Emerging Nanophotonic Technology," In ISCA, 2008.

[5] Xiang Zhang and Ahmed Louri, "A Multilayer Nanophotonic Interconnection Network for On-Chip Many-core Communications,” In DAC, 2010.

[6] George Kurian, et al., "ATAC: a 1000-core cache-coherent processor with onchip optical network," In $P A C T, 2010$.
[7] Yan Pan, et al., "Firefly: Illuminating Future Network-on-Chip with Nanophotonics," In ISCA, 2009.

[8] Sébastien Le Beux, et al., "Optical Ring Network-on-Chip (ORNoC): Architecture and design methodology," In DATE, 2011.

[9] Ajay Josh, et al. "Silicon-Photonic Clos Networks for Global On-Chip Communication," In NoCS, 2009.

[10] Sébastien Le Beux, et al., "CHAMELEON: CHANNEL Efficient Optical Network-on-Chip," In DATE, 2014.

[11] Xiaowen Wu, et al., "SUOR: Sectioned Undirectional Optical Ring for Chip Multiprocessor," ACM J. Emerging Technologies in Computing Systems, 2014.

[12] Cheng Li, et al., "LumiNOC: A Power-Efficient, High-Performance, Photonic Network-on-Chip for Future Parallel Architectures," In PACT, 2012.

[13] Yaoyao Ye, et al, "A Torus-Based Hierarchical Optical-Electronic Network-onChip for Multiprocessor System-on-Chip," ACM Journal on Emerging Technologies in Computing Systems (JETC), 8 (1), 5:1-5:26, 2012.

[14] Qianfan Xu, et al., "12.5 Gbit/s carrier-injection-based silicon micro-ring silicon modulators," Optics Express, 15(2), 430-436, 2007. 